\title{
Permanence and periodic solution for a modified Leslie-Gower type predator-prey model with diffusion and non constant coefficients
}

\author{
A. Moussaoui ${ }^{1}$, M. A. Aziz Alaoui ${ }^{2}$, R. Yafia ${ }^{3}$ \\ ${ }^{1}$ Department of Mathematics, University of Tlemcen, Tlemcen, Algeria \\ moussaoui.ali@gmail.com \\ ${ }^{2}$ Normandie University, Le Havre, France \\ ULH, LMAH, FR CNRS 3335, Le Havre, France \\ aziz.alaoui@univ-lehavre.fr \\ ${ }^{3}$ Université Ibn Zohr, Faculté Polydisciplinaire, Ouarzazate, Morocco \\ yafia@yahoo.fr
}

Received: 5 March 2017, accepted: 10 July 2017, published: 19 July 2017

\begin{abstract}
In this paper we study a predator-prey system, modeling the interaction of two species with diffusion and $T$-periodic environmental parameters. It is a Leslie-Gower type predator-prey model with Holling-type-II functional response. We establish some sufficient conditions for the ultimate boundedness of solutions and permanence of this system. By constructing an appropriate auxiliary function, the conditions for the existence of a unique globally stable positive periodic solution are also obtained. Numerical simulations are presented to illustrate the results.
\end{abstract}

Keywords-Reaction-diffusion equations, Predatorprey model, Functional response, Permanence.

\section{INTRODUCTION AND MATHEMATICAL MODEL}

The dynamical properties of the predator-prey models can be used to analyze the relations between the prey and predator and to predicate whether they can coexist. As we known, one of the earliest and also the best known predatorprey models is the Leslie-Gower model [16], [17], which is a modificiation of the Lotka-Volterra model [22]. The Leslie-Gower type model can be described by the following autonomous bidimensional system [16], [17]

$$
\left\{\begin{array}{l}
\frac{d u}{d t}=u(a-b u)-\alpha u v \\
\frac{d v}{d t}=v\left(c-\frac{\beta v}{u}\right)
\end{array}\right.
$$

where $u$ is the population of the prey and $v$ is the population of the predator. In (1) we assume the prey grows logistically with carrying capacity $K=\frac{a}{b}$ and intrinsic growth rate $a$ in the absence of predation. The predation is assumed to be proportional to the population size of the prey. The predator grows logistically with intrinsic growth rate $c$ and carrying capacity $\frac{c}{\beta} u(t)$ proportional to the population size of prey (or prey abundance). 
The parameter $\beta$ is a measure of the food quality that the prey provides for conversion into predator birth. The stability of the interior equilibrium is studied in [25] by numerical methods. Lindstrom [18] investigated the nonexistence, existence and limit cycles. Hsu and Huang [13] prove that all the solutions are bounded and positive if their initial values are in the first quadrant, and study the globally asymptotical stability of the interior equilibrium using Liapunov function and LaSalle's invariance principle.

Aziz-Alaoui and Daher Okiye [3] argued that a suitable predator-prey model should incorporate some kind of functional response, while the predator species could have other food resource. Basing on those assumption, they proposed a predator-prey model with modified Leslie-Gower and Holling-type II schemes [12] as follow:

$$
\left\{\begin{aligned}
\frac{d u}{d t} & =u(a-b u)-\frac{\alpha u v}{u+k_{1}} \\
\frac{d v}{d t} & =v\left(c-\frac{\beta v}{u+k_{2}}\right)
\end{aligned}\right.
$$

where $k_{1}$ is the half-saturation constant in the Holling-type II functional response and $k_{2}$ is a measure of alternative prey densities in the environment, allowing the predator to persist when the prey population disappears. The authors investigated the boundedness and global stability of the system (2). Nindjin et al. [23] further incorporated the time delay to the system considered in [3], and they showed that time delay plays important role on the dynamic behaviors of the system. Yafia et al. [27] studied the limit cycle bifurcated from time delay. For more works on Leslie-Gower predator-prey model, one could refer to [1], [4], [7], [8], [14], [26], [28], [29] and the references cited therein.

To achieve further understanding it is now essential to consider more general and hence more "difficult" models. We will focus here on the case in which the biological or environmental parameters are time-periodic, and will assume that the species are free to move at random throughout some bounded habitat. Under these assumptions, we model the species interaction via a system of reaction-diffusion equations of the form

$$
\left\{\begin{array}{l}
\frac{\partial u}{\partial t}-d_{1} \Delta u=u(a(t, x)-b(t, x) u)-\frac{\alpha(t, x) u v}{u+k_{1}(t, x)} \\
\frac{\partial v}{\partial t}-d_{2} \Delta v=v\left(c(t, x)-\frac{\beta(t, x) v}{u+k_{2}(t, x)}\right)
\end{array}\right.
$$

where the function $u(t, x)$ and $v(t, x)$ determine the densities of prey and predator, respectively, at a point $x$ and time $t$. Here the equations are assumed to be satisfied in a cylinder $x \in \bar{\Omega}, 0<t<\infty$, where $\Omega$ is an open, bounded, smooth domain in $\mathbb{R}^{n}$. These equations are supplemented with homogeneous Neumann boundary conditions

$$
\frac{\partial u}{\partial n}=\frac{\partial v}{\partial n}=0 \text { on } \partial \Omega \times(0, \infty) .
$$

where $n$ is the outward unit vector of the boundary $\partial \Omega$ which we assume is smooth, and the following nonnegative initial values

$$
u(0, x)=u_{0}(x) \geq 0, \quad v(0, x)=v_{0}(x) \geq 0 \text { in } \Omega .
$$

The various coefficients on the right-hand side depend on both $t$ and $x$ modelling the fact that effects vary in both time and space. The periodicity of coefficients models seasonal fluctuations. $d_{1}$ and $d_{2}$, are positive diffusion coefficients reflecting the non-homogeneous dispersion of populations. Many authors studied the qualitative properties of this system, but for the case for which the parameters are constant, using Neumann or Dirichlet boundary conditions, see [8], [24], [27], [29].

Motivated by the papers mentioned above, we deal here with the permanence and existence of periodic solutions of the diffusive system (3).

The content of this paper is as follows. In section 2 , we give conditions for the ultimate boundedness of solutions and permanence of the system. In Section 3, we establish conditions for the existence of a unique periodic solution of the system. $\mathrm{Nu}$ merical simulations are presented in Section 4 to illustrate the feasibility of our results. 
A. Moussaoui et al., Permanence and periodic solution for a modified Leslie-Gower type ...

\section{Boundedness AND PERMANENCE}

We analyze the permanence (dissipation and persistence) of system (3) with non-negative initial functions, this ensures the long-term survival (i.e., will not vanish in time) of all components of system (3), under some conditions. We first recall a well known result on the logistic equation.

Lemma 2.1: [30]. Assume that $u(t, x)$ is defined by

$$
\left\{\begin{array}{l}
\frac{\partial u}{\partial t}=d_{1} \Delta u+r u\left(1-\frac{u}{K}\right), x \in \Omega, t>0, \\
\frac{\partial u}{\partial n}=0, \quad x \in \partial \Omega, \quad t>0 \\
u(x, 0)=u_{0}(x)>0, \quad x \in \Omega,
\end{array}\right.
$$

then, $\lim _{t \rightarrow \infty} u(t, x)=K$.

Throughout the paper we always assume that:

$(\mathbf{H})$ : Functions $a(t, x), b(t, x), c(t, x), \alpha(t, x)$, $\beta(t, x)$ and $k_{i}(t, x),(i=1,2)$ are bounded positive-valued functions on $\mathbb{R}_{+} \times \bar{\Omega}$ continuously differentiable in $t$ and $x$, and are periodic in $t$ with a period $T>0$.

For a bounded function $\phi(t, x)$, we denote

$$
\begin{aligned}
\phi^{m} & =\inf _{(t, x) \in \mathbb{R}_{+} \times \bar{\Omega}} \phi(t, x), \\
\phi^{M} & =\sup _{(t, x) \in \mathbb{R}_{+} \times \bar{\Omega}} \phi(t, x) .
\end{aligned}
$$

\section{A. Dissipation}

Proposition 2.2: All the solutions of (3) initiated in the positive octant are nonnegative and satisfy

$$
\begin{aligned}
& \limsup _{t \rightarrow+\infty} \max _{x \in \bar{\Omega}} u(t, x) \leq \frac{a^{M}}{b^{m}}, \\
& \limsup _{t \rightarrow+\infty} \max _{x \in \bar{\Omega}} v(t, x) \leq \frac{c^{M}}{\beta^{m}}\left(\frac{a^{M}}{b^{m}}+k_{2}^{M}\right) .
\end{aligned}
$$

\section{Proof}

The nonnegativity of the solutions of (3) is obvious since the initial value is nonnegative. We consider now the second part of the theorem. For convenience, we denote $a=a(t, x)$, and similar meaning to $b, c, \alpha, \beta, k_{1}$ and $k_{2}$. From the first equation of system (3), we have

$$
\begin{aligned}
\frac{\partial u}{\partial t} & =d_{1} \Delta u+u(a-b u)-\frac{\alpha u v}{u+k_{1}}, \\
& \leq d_{1} \Delta u+u\left(a^{M}-b^{m} u\right)
\end{aligned}
$$

From the comparison principle of the parabolic equations [9], [11], it is easy to verify that $u(t, x) \leq \bar{u}(t)$, where $\bar{u}(t)$ is the spatially homogeneous solutions of

$$
\left\{\begin{array}{l}
\frac{\partial u}{\partial t}=d_{1} \Delta u+u\left(a^{M}-b^{m} u\right), \quad x \in \Omega, t>0 \\
\frac{\partial u}{\partial n}=0, \quad x \in \partial \Omega, t>0 \\
u(0, x)=u_{*}
\end{array}\right.
$$

where $u_{*}=\max _{x \in \bar{\Omega}} u(0, x)$. This implies, by using Lemma 2.1, that

$$
\limsup _{t \rightarrow+\infty} \max _{x \in \bar{\Omega}} u(t, x) \leq \frac{a^{M}}{b^{m}} .
$$

Then, for $\varepsilon>0$ there exists $T_{1}>0$ such that

$$
u(t, x) \leq \eta_{1} \text { for } t>T_{1},
$$

where $\eta_{1}=\frac{a^{M}}{b^{m}}+\varepsilon$.

Therefore, from the second equation of system (3) and (7) and using the same reasoning, we have

$$
\begin{aligned}
\frac{\partial v}{\partial t} & =d_{2} \Delta v+v\left(c-\frac{\beta v}{u+k_{2}}\right) \\
& \leq d_{2} \Delta v+v\left(c^{M}-\frac{\beta^{m} v}{\eta_{1}+k_{2}^{M}}\right)
\end{aligned}
$$

for $t>T_{1}$. Hence there exists $T_{2}>T_{1}$ such that for any $t>T_{2}$

$$
v(t, x) \leq \eta_{2}
$$

where $\eta_{2}=\frac{c^{M}}{\beta^{m}}\left(\frac{a^{M}}{b^{m}}+\varepsilon+k_{2}^{M}\right)+\varepsilon$, which implies

$$
\limsup _{t \rightarrow+\infty} \max _{x \in \bar{\Omega}} v(t, x) \leq \frac{c^{M}}{\beta^{m}}\left(\frac{a^{M}}{b^{m}}+k_{2}^{M}\right) .
$$

Therefore, any positive solution of system (3) is ultimately bounded, which completes the proof. 
A. Moussaoui et al., Permanence and periodic solution for a modified Leslie-Gower type ...

\section{B. Persistence}

Definition 2.3: [5], [6] System (3) is said to be persistent if for any positive initial data $\left(u_{0}(x), v_{0}(x)\right)$, there exist positive constants $\xi_{1}=\xi_{1}\left(u_{0}, v_{0}\right), \xi_{2}=\xi_{2}\left(u_{0}, v_{0}\right)$, such that the solution $(u(t, x), v(t, x))$ of (3) satisfies

$\liminf _{t \rightarrow+\infty} \min _{x \in \bar{\Omega}} u(t, x) \geq \xi_{1}, \quad \liminf _{t \rightarrow+\infty} \min _{x \in \bar{\Omega}} v(t, x) \geq \xi_{2}$

Proposition 2.4: Assume that

$$
a^{m} k_{1}^{m} \beta^{m}>\alpha^{M} c^{M}\left(\frac{a^{M}}{b^{m}}+k_{2}^{M}\right)
$$

then system (3) is persistent.

\section{Proof}

From (3), (7) and (8), it follows that for $t \geq T_{2}$,

$$
\begin{aligned}
\frac{\partial u}{\partial t}= & d_{1} \Delta u+u(a-b u)-\frac{\alpha u v}{u+k_{1}} \\
& \geq d_{1} \Delta u+u\left(a^{m}-b^{M} u\right)-\frac{\alpha^{M} \eta_{2} u}{k_{1}^{m}} \\
& =d_{1} \Delta u+u\left(a^{m}-\frac{\alpha^{M} \eta_{2}}{k_{1}^{m}}-b^{M} u\right)
\end{aligned}
$$

then from the comparison principle of the parabolic equations, it is easy to verify that $u(t, x) \geq \bar{u}(t)$, where $\bar{u}(t)$ is the spatially homogeneous solutions of

$$
\left\{\begin{array}{l}
\frac{\partial u}{\partial t}=d_{1} \Delta u+u\left(a^{m}-\frac{\alpha^{M} \eta_{2}}{k_{1}^{m}}-b^{M} u\right) \\
x \in \Omega, t>0 \\
\frac{\partial u}{\partial n}=0, x \in \partial \Omega, \quad t>0 \\
u(x, 0)=u^{*}
\end{array}\right.
$$

where $u^{*}=\min _{x \in \bar{\Omega}} u(0, x)$. Thanks to lemma 2.1, we obtain,

$$
\liminf _{t \rightarrow+\infty} \min _{x \in \bar{\Omega}} u(t, x) \geq \frac{1}{b^{M}}\left(a^{m}-\frac{\alpha^{M} \eta_{2}}{k_{1}^{m}}\right) .
$$

Hence, there exists $T_{3}>T_{2}$ such that for any $t>T_{3}$,

$$
u(t, x) \geq \xi_{1}
$$

where,

$$
\xi_{1}=\frac{1}{b^{M}}\left(a^{m}-\frac{\alpha^{M} \eta_{2}}{k_{1}^{m}}-\varepsilon\right) .
$$

From the predator equation, it follows that

$$
\frac{\partial v}{\partial t}=d_{2} \Delta v+v\left(c-\frac{\beta v}{u+k_{2}}\right)
$$

$$
\geq d_{2} \Delta v+v\left(c^{m}-\frac{\beta^{M} v}{k_{2}^{m}}\right) .
$$

Hence, there exists $T_{4}>0$ such that for any $t>T_{4}$

$$
v(t, x)>\xi_{2},
$$

where

$$
\xi_{2}=\frac{c^{m} k_{2}^{m}}{\beta^{M}}-\varepsilon
$$

Therefore, from (11) and (12), we obtain,

$$
\begin{aligned}
& \liminf _{t \rightarrow+\infty} \min _{x \in \bar{\Omega}} u(t, x) \\
& \quad \geq \frac{1}{b^{M}}\left(a^{m}-\frac{\frac{\alpha^{M} c^{M}}{\beta^{m}}\left(\frac{a^{M}}{b^{m}}+k_{2}^{M}\right)}{k_{1}^{m}}\right), \\
& \liminf _{t \rightarrow+\infty} \min _{x \in \bar{\Omega}} v(t, x) \geq \frac{c^{m} k_{2}^{m}}{\beta^{M}} .
\end{aligned}
$$

Thus, system (3) is persistent, which completes the proof of Proposition 2.4. A direct application of Proposition 2.2 and Proposition 2.4 gives the following result.

Proposition 2.5: (Permanence) If condition 9) holds, there exist positive constants $0<\zeta<\eta$, such that,

$$
\zeta \leq \liminf _{t \rightarrow \infty} \min _{x \in \bar{\Omega}} u(t, x) \leq \limsup _{t \rightarrow \infty} \max _{x \in \bar{\Omega}} u(t, x) \leq \eta
$$

$\zeta \leq \liminf _{t \rightarrow \infty} \min _{x \in \bar{\Omega}} v(t, x) \leq \limsup _{t \rightarrow \infty} \max _{x \in \bar{\Omega}} v(t, x) \leq \eta$

That is, model (3) is permanent. 
A. Moussaoui et al., Permanence and periodic solution for a modified Leslie-Gower type ...

\section{PERIOdiC SOLUTIONS}

A very basic and important problem in the study of a population growth models with periodic environment is the global existence and stability of positive periodic solutions, which plays a similar role as a globally stable equilibrium for autonomous models [5], [11], [15], [19], [21]. In this section, we derive sufficient conditions that guarantee existence, uniqueness and global stability of a $T$-periodic positive solution of system (3). For this aim, we consider the matrix $M$, which reads as:

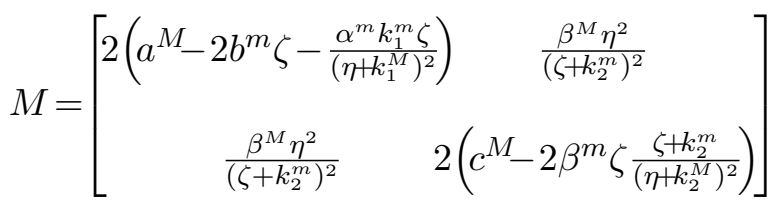

where $\zeta$ and $\eta$ are the bounds of any non-zero arbitrary solution of system (3), initialing with non-negative function, given by Proposition 2.5 .

Proposition 3.1: Assume that condition (9) holds, that is system (3) is permanent, if

$$
\mu(M)<0,
$$

where $\mu(M)$ is the maximal eigenvalue of the matrix $M$. Then, system (3) has a unique globally asymptotic stable strictly positive $T$-periodic solution.

\section{Proof}

Let $\left(u_{1}(t, x), v_{1}(t, x)\right)$ and $\left(u_{2}(t, x), v_{2}(t, x)\right)$ be two solutions of system (3), by Proposition 2.5. these solutions are bounded by constants $\zeta$ and $\eta$, where $\zeta=\min \left\{\xi_{1}, \xi_{2}\right\}$ and $\eta=\max \left\{\eta_{1}, \eta_{2}\right\}$, defined in section 2. Consider the function

$$
U(t)=\int_{\Omega}\left(\left(u_{1}(t, x)-u_{2}(t, x)\right)^{2}+\left(v_{1}(t, x)-v_{2}(t, x)\right)^{2}\right) d x
$$

One has,

$$
\begin{gathered}
\frac{d U(t)}{d t}=2 \int_{\Omega}\left(u_{1}-u_{2}\right)\left(\frac{\partial u_{1}}{\partial t}-\frac{\partial u_{2}}{\partial t}\right) d x \\
+2 \int_{\Omega}\left(v_{1}-v_{2}\right)\left(\frac{\partial v_{1}}{\partial t}-\frac{\partial v_{2}}{\partial t}\right) d x \\
=2 d_{1} \int_{\Omega}\left(u_{1}-u_{2}\right) \Delta\left(u_{1}-u_{2}\right) d x \\
+2 d_{2} \int_{\Omega}\left(v_{1}-v_{2}\right) \Delta\left(v_{1}-v_{2}\right) d x
\end{gathered}
$$

$$
\begin{aligned}
& +2 \int_{\Omega}\left(u_{1}-u_{2}\right) \\
& {\left[\left(u_{1}\left(a-b u_{1}\right)-\frac{\alpha u_{1} v_{1}}{u_{1}+k_{1}}\right)-\left(u_{2}\left(a-b u_{2}\right)-\frac{\alpha u_{2} v_{2}}{u_{2}+k_{1}}\right)\right] d x} \\
& +2 \int_{\Omega}\left(v_{1}-v_{2}\right)\left[\left(v_{1}\left(c-\frac{\beta v_{1}}{u_{1}+k_{2}}\right)-\left(v_{2}\left(c-\frac{\beta v_{2}}{u_{2}+k_{2}}\right)\right] d x\right.\right. \\
& :=I_{1}+I_{2}+I_{3}+I_{4}
\end{aligned}
$$

It follows from the boundary condition in $(3)$ that

$$
\begin{aligned}
& I_{1}+I_{2}= 2 d_{1} \int_{\partial \Omega}\left(u_{1}-u_{2}\right) \nabla\left(u_{1}-u_{2}\right) d \eta \\
&-2 d_{1} \int_{\Omega}\left(\nabla\left(u_{1}-u_{2}\right)\right)^{2} d x \\
&+2 d_{2} \int_{\partial \Omega}\left(v_{1}-v_{2}\right) \nabla\left(v_{1}-v_{2}\right) d \eta \\
&-2 d_{2} \int_{\Omega}\left(\nabla\left(v_{1}-v_{2}\right)\right)^{2} d x \\
&=-2 d_{1} \int_{\Omega}\left(\nabla\left(u_{1}-u_{2}\right)\right)^{2} d x-2 d_{2} \int_{\Omega}\left(\nabla\left(v_{1}-v_{2}\right)\right)^{2} d x .
\end{aligned}
$$$$
\leq 0 \text {. }
$$

For the third and fourth term in (17), we have

$$
\begin{aligned}
& I_{3}+I_{4}=2 \int_{\Omega}\left(u_{1}-u_{2}\right)\left[\left(\left(u_{1}-u_{2}\right)\left(a-b\left(u_{1}+u_{2}\right)\right)\right.\right. \\
& \left.-\alpha \frac{u_{1} u_{2}\left(v_{1}-v_{2}\right)+k_{1}\left(u_{1} v_{1}-u_{2} v_{2}\right.}{\left(u_{1}+k_{1}\right)\left(u_{2}+k_{1}\right)}\right] d x \\
& \quad+2 \int_{\Omega}\left(v_{1}-v_{2}\right)\left[c\left(v_{1}-v_{2}\right)\right. \\
& \left.-\beta \frac{\left(v_{1}^{2} u_{2}-v_{2}^{2} u_{1}\right)+k_{2}\left(v_{1}-v_{2}\right)\left(v_{1}+v_{2}\right)}{\left(u_{1}+k_{2}\right)\left(u_{2}+k_{2}\right)} d x\right] .
\end{aligned}
$$

Note that

$v_{1}^{2} u_{2}-v_{2}^{2} u_{1}=\left(v_{1}-v_{2}\right)\left(v_{1} u_{2}+v_{2} u_{1}\right)-v_{1} v_{2}\left(u_{1}-u_{2}\right)$

and

$$
u_{1} v_{1}-u_{2} v_{2}=u_{1}\left(v_{1}-v_{2}\right)+v_{2}\left(u_{1}-u_{2}\right) .
$$

Therefore

$$
\begin{aligned}
& I_{3}+I_{4}= \\
& 2 \int_{\Omega}\left(u_{1}-u_{2}\right)^{2}\left[a-b\left(u_{1}+u_{2}\right)-\frac{\alpha k_{1} v_{2}}{\left(u_{1}+k_{1}\right)\left(u_{2}+k_{1}\right)}\right] d x \\
& +2 \int_{\Omega}\left(v_{1}-v_{2}\right)^{2}\left[c-\beta \frac{\left(v_{1} u_{2}+v_{2} u_{1}\right)+k_{2}\left(v_{1}+v_{2}\right)}{\left(u_{1}+k_{2}\right)\left(u_{2}+k_{2}\right)} d x\right]
\end{aligned}
$$


A. Moussaoui et al., Permanence and periodic solution for a modified Leslie-Gower type ...

$$
\begin{aligned}
& +2 \int_{\Omega}\left(u_{1}-u_{2}\right)\left(v_{1}-v_{2}\right) \\
& {\left[\frac{\beta v_{1} v_{2}}{\left(u_{1}+k_{2}\right)\left(u_{2}+k_{2}\right)}-\frac{\alpha u_{1}}{\left(u_{1}+k_{1}\right)}\right] d x} \\
& \leq 2 \int_{\Omega}\left(u_{1}-u_{2}\right)^{2}\left[a^{M}-2 b^{m} \zeta-\frac{\alpha^{m} k_{1}^{m} \zeta}{\left(\eta+k_{1}^{M}\right)^{2}}\right] d x \\
& +2 \int_{\Omega}\left(v_{1}-v_{2}\right)^{2}\left[c^{M}-2 \beta^{m} \zeta \frac{\zeta+k_{2}^{m}}{\left(\eta+k_{2}^{M}\right)^{2}}\right] d x \\
& +2 \int_{\Omega}\left|\left(u_{1}-u_{2}\right)\left(v_{1}-v_{2}\right)\right| \frac{\beta^{M} \eta^{2}}{\left(\zeta+k_{2}^{m}\right)^{2}} d x \\
& \leq \mu(M) \int_{\Omega}\left[\left(u_{1}-u_{2}\right)^{2}+\left(v_{1}-v_{2}\right)^{2}\right] d x .
\end{aligned}
$$

Using (15) yields,

$$
U(t) \leq U(0) e^{\mu(M) t} \rightarrow 0 \text { as } t \rightarrow \infty
$$

Thus, we have proved that $\left\|u_{1}(t, x)-u_{2}(t, x)\right\| \rightarrow$ 0 and $\left\|v_{1}(t, x)-v_{2}(t, x)\right\| \rightarrow 0$ as $t \rightarrow \infty$, where $\|$.$\| denotes the norm of the space L_{2}(\Omega)$.

Let $p>n$ a positive integer and $w\left(t, w_{0}\right)=$ $\left(u\left(t, x, u_{0}, v_{0}\right), v\left(t, x, u_{0}, v_{0}\right)\right)$. By applying exactly the same reasoning as in [2], we prove that for some $\gamma \in\left(\frac{1}{2}+\frac{n}{2 p}, 1\right)$, the solution $\left\{w\left(t, w_{0}\right)\right\}$ is relatively compact in the space $C^{1+\theta}\left(\bar{\Omega}, \mathbb{R}^{2}\right)$, for $0<\theta<2 \gamma-1-n / p$. Therefore,

$$
\begin{aligned}
& \lim _{t \rightarrow \infty} \sup _{x \in \Omega}\left|u_{1}(t, x)-u_{2}(t, x)\right|=0, \\
& \lim _{t \rightarrow \infty} \sup _{x \in \Omega}\left|v_{1}(t, x)-v_{2}(t, x)\right|=0 .
\end{aligned}
$$

Now we consider the sequence

$$
\left(u\left(k T, x, u_{0}, v_{0}\right), v\left(k T, x, u_{0}, v_{0}\right)\right)=w\left(k T, w_{0}\right) .
$$

Then, $\left\{w\left(k T, w_{0}\right), k \in \mathbb{N}\right\}$ is compact in the space $C(\bar{\Omega}) \times C(\bar{\Omega})$. Let $\bar{\omega}$ be a limit point of this sequence, then $w(T, \bar{w})=\bar{w}$. Indeed, it follows, from $w\left(T, w\left(k_{n} T, w_{0}\right)\right)=w\left(k_{n} T, w\left(T, w_{0}\right)\right)$ and $\omega\left(k_{n} T, w\left(T, \omega_{0}\right)\right)-w\left(k_{n} T, \omega_{0}\right) \rightarrow 0$ as $k_{n} \rightarrow \infty$, that

$$
\begin{gathered}
\|w(T, \bar{w})-\bar{w}\|_{C} \leq\left\|w(T, \bar{w})-w\left(T, w\left(k_{n} T, w_{0}\right)\right)\right\|_{C} \\
+\left\|w\left(T, w\left(k_{n} T, w_{0}\right)\right)-w\left(k_{n} T, w_{0}\right)\right\|_{C} \\
+\left\|w\left(k_{n} T, w_{0}\right)-\bar{w}\right\|_{C} \rightarrow 0 \text { as } n \rightarrow \infty .
\end{gathered}
$$

The sequence $\left\{w\left(k T, w_{0}\right), k \in N\right\}$ has a unique limit point, otherwise, there are two limit points $\bar{w}=\lim _{t \rightarrow \infty} w\left(k_{n} T, w_{0}\right)$ and $\hat{w}=\lim _{t \rightarrow \infty} w\left(k_{n} T, w_{0}\right)$ But, thanks to (19) and $\hat{w}=w\left(\stackrel{t \rightarrow \infty}{k_{n} T, \hat{w}}\right)$, we get

$$
\begin{aligned}
& \|\bar{w}-\hat{w}\|_{C} \leq \\
& \left\|\bar{w}-w\left(k_{n} T, w_{0}\right)\right\|_{C}+\left\|w\left(k_{n} T, w_{0}\right)-\hat{w}\right\|_{C} \rightarrow 0 \\
& \quad \text { as } n \rightarrow \infty .
\end{aligned}
$$

Thus, $\bar{w}=\hat{w}$. Hence, the solution $(u(t, x, \bar{u}, \bar{v}), v(t, x, \bar{u}, \bar{v}))$ is the unique periodic solution of system (3). Finally, due to (19), we conclude that this periodic solution is globally asymptotically stable.

\section{NUMERICAL SIMULATIONS}

In this section, numerical simulations for a given parameters range of system (3) are done to support our analytical results obtained in Sections 3 and 4. We consider system (3) with $d_{1}=$ $0.5, d_{2}=0.8, a=2+0.5 \sin (2 \pi t), \quad b=4+$ $0.5 \sin (2 \pi t), \quad \alpha=0.03+0.02 \sin (2 \pi t), \quad k_{1}=$ $1+0.2 \sin (2 \pi t), \quad k_{2}=1+0.5 \sin (2 \pi t), \quad c=$ $1+0.6 \sin (2 \pi t)$ and $\beta=1+0.8 \sin (2 \pi t)$.

Obviously, all the parameters have a common period $T=1$ in $t$, By a direct computation, we can prove that all conditions in proposition 4 are satisfied. Then, system (3) has a unique positive 1-periodic solution $u(t, x), v(t, x)$ which is globally asymptotically stable. By applying Matlab to simulate, we can obtain Figures 1-4. From these figures, we see that system (3) is permanent and has positive periodic solution.

\section{CONCLUSION}

The interacting species play important roles in real ecosystem. In this paper, we have studied time-periodic Leslie-Gower type predatorprey model with diffusion and Holling-type-II functional response whose growth rates and interaction rates are periodic functions of time. We have obtained sufficient conditions for the persistence of (3) in Proposition 2.4. The conditions are given in term of parameters of the model. Biologically speaking, we may expect the coexistence when the predator growth rate is sufficiently small, or if the predation rate $\alpha$ is small enough. 


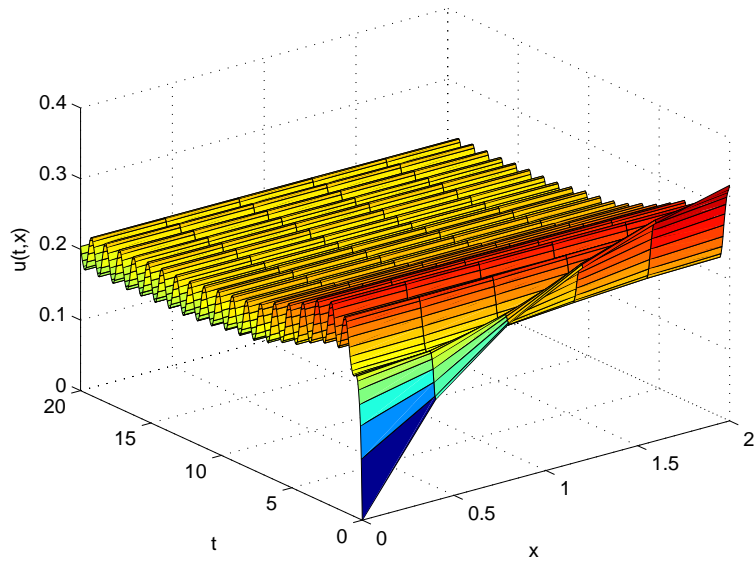

Fig. 1. Periodic prey solution with respect to the time and space variables.

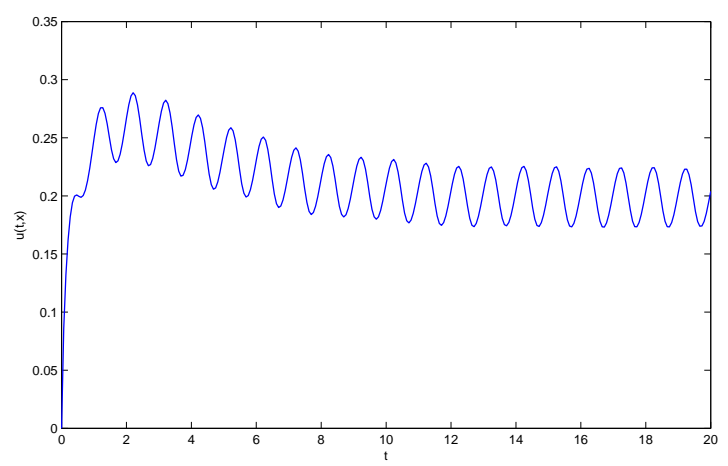

Fig. 2. The section of Fig. 1 with $x=0$.

Next, we have investigated sufficient conditions which ensure the existence of positive $T$-periodic solutions of (3) in Proposition 3.1. The conditions are given in term of the largest eigenvalue of certain matrix. Our study demonstrates how parameters of the model which are not constant but vary in response to environmental fluctuations, influence a species prosperity, and gives some valuable suggestions for saving the two species and regulating populations when the ecological and environmental parameters are affected by periodic factors such as the season switching. Numerical simulations are carried out to support our theoretical results.

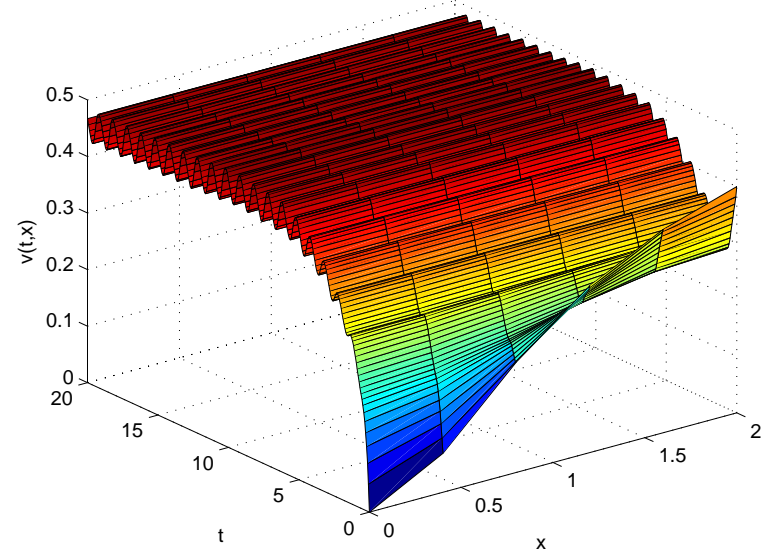

Fig. 3. Periodic prey solution with respect to the time and space variables.

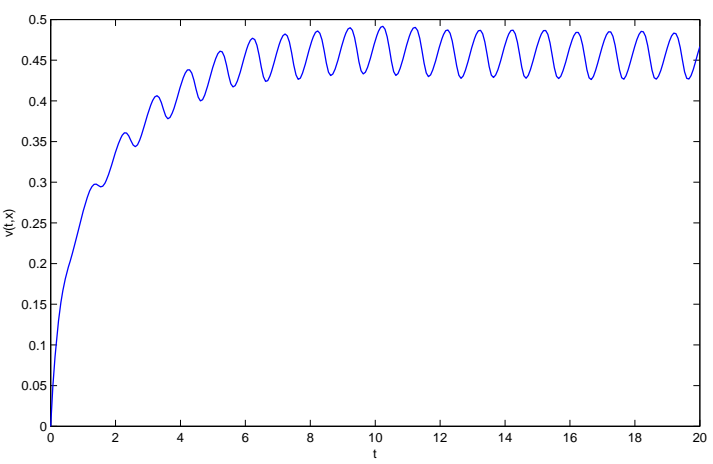

Fig. 4. The section of Fig. 3 with $x=0$.

\section{Acknowledgments}

The authors would like to thank the editor and anonymous referees for their careful reading of the manuscript and valuable suggestions to improve the quality of this work.

\section{REFERENCES}

[1] P. Aguirre, E. Gonzalez-Olivares, E. Saez, Two limit cycles in a Leslie-Gower predator-prey model with additive Allee effect, Nonlinear Anal. Real World Appl., 10, 14011416, 2009.

[2] M. U. Akhmet, M. Beklioglub, T. Ergenca, V. I. Tkachenkoc, An impulsive ratio-dependent predator-prey system with diffusion, Nonlinear Analysis: Real World Applications 7, 1255-1267, 2006. 
[3] M. A. Aziz-Alaoui and M. Daher Okiye, Boudedness and global stability for a predator-prey model with modified Leslie-Gower and Holling type II schemes, Applied Math. Lets. 16, 1069-1075, 2003.

[4] M. A. Aziz-Alaoui, Study of a Leslie-Gower-type tritrophic population model, Chaos, Solitons and Fractals, 14, 1275-1293, 2002.

[5] R. S. Cantrell, C. Cosner, Spatial Ecology via ReactionDiffusion Equations, Wiley Ser. Math. Comput. Biol. John Wiley-Sons, Ltd. 2003.

[6] R. S. Cantrell, C. Cosner, Diffusion models for population dynamics incorporating individual behavior at boundaries: Applications to refuge design, Theoretical Pop. Biology, 55, 189-207, 1999.

[7] B. Chen, M. Wang, Qualitative analysis for a diffusive predator-prey model, Comput. Math. Appl., 55, 339-355, 2008.

[8] L. J Chen, F. D. Chen, Global stability of a Leslie-Gower predator-prey model with feedback controls, Appl. Math. Lett., 22, 1330-1334, 2009.

[9] A. Friedman, Partial Differential Equations of Parabolic Type, Prentice-Hall, Inc. Englewood Cliffs, N.J, 1964.

H. I. Freedman, R. M. Mathsen, Persistence in predatorprey systems with ratio-dependence predation influence, Bull. Math. Biol., 55, 817-827, 1993.

[10] R. Gupta R, P. Chandra, Bifurcation analysis of modified Leslie-Gower predator-prey model with MichaelisMenten type prey harvesting. J Math Anal Appl., 398 (1): 278-295, 2013.

[11] P. Hess, Periodic-parabolic boundary value problems and positivity, Pitman Research Notes in Mathematics Series, Beijing, 1991.

[12] C. S. Holling, The components of predation as revealed by a study of small mammal predation of the European pine sawfly, Can. Entomol., 91, 293-320, 1959.

[13] S. B. Hsu and T. W. Huang, Global stability for a class of predator-prey systems, SIAM J. Appl. Math.,55, 763783, 1995.

[14] A. Korobeinikov, A Lyapunov function for Leslie-Gower predator-prey models, Appl. Math. Lett., 14, 697-699, 2001.

[15] A. C. Lazer, Some remarks on periodic solutions of parabolic differential equations, Dynamical Systems., II Proceedings of the Second International Symposium held at the University of Florida, Gainesville, Fla., February 25-27, 1981, A. R. Bednarek and L. Cesari, eds., Academic Press, Kaunas, 227-246, 1982.

[16] P. H. Leslie, Some further notes on the use of matrices in population mathematics, Biometrika, 35, 213-245, 1948.
[17] P. H. Leslie, A stochastic model for studying the properties of certain biological systems by numerical methods, Biometrika, 45(1-2):16-31, 1958.

[18] T. Lindstrom, Qualitative analysis of a predator-prey system with limit cycles, J. Math. Biol., 31, 541-561, 1993.

[19] X. Liu, Permanence and periodic solutions for a diffusive ratio-dependent predator-prey system, Applied Mathematical Modelling, 33, 683-691, 2009.

[20] R. M. May, Stability and complexity in model ecosystem, Princeton Uni- versity Press, Princeton, NJ 1974.

[21] A. Moussaoui, A reaction-diffusion equations modelling the effect of fluctuating water levels on prey-predator interactions, Applied Mathematics and Computation 268, 1110-1121, 2015.

[22] J. D. Murray, Mathematical Biology, Springer-Verlag, Berlin, 1989.

[23] A. F. Nindjin, M. A. Aziz-Alaoui and M. Cadivel, Analysis of a predator-prey model with modified LeslieGower and Holling-type II schemes with time delay, Nonlinear Anal., Real World Appl., 7, 1104-1118, 2006.

[24] R. Peng, M. Wang, On multiplicity and stability of positive solutions of a diffusive prey-predator model, Math. Anal. Appl., 316, 256-268, 2006.

[25] E. C. Pielou, An Introduction to Mathematical Ecology, 2nd edition, John Wiley \& Sons, New York, 1977.

[26] R. Yafia , F. Adnani, H. Talibi Alaoui, Stability of limit cycle in a predator-prey model with modified LeslieGower and Holling-type II schemes with time delay. Appl Math Sci., 1(3) : 119-131, 2007.

[27] R. Yafia, M. A. Aziz-Alaoui, Existence of periodic travelling waves solutions in predator prey model with diffusion, Applied Mathematical Modelling, 37, 36353644, 2013.

[28] W. Yang, Global asymptotical stability and persistent property for a diffusive predator-prey system with modified Leslie-Gower functional response, Nonlinear Analysis: Real World Applications, 14, 1323-1330, 2013.

[29] W. S. Yang,Y. Q. Li, Dynamics of a diffusive predatorprey model with modified Leslie-Gower and Holling-type III schemes, Comput. Math. Appl., 65, 1727-1737, 2013.

[30] Q. Ye, Introduction to Reaction-Diffusion Equations, Science Press, Beijing, 2013.

[31] Q. Yue, Dynamics of a modified Leslie-Gower predator-prey model with Holling-type II schemes and a prey refuge, SpringerPlus 5: 461, (2016). DOI 10.1186/s40064-016-2087-7. 